\title{
Gut microbiome and liver diseases
}

\author{
Herbert Tilg, ${ }^{1}$ Patrice D Cani, ${ }^{2}$ Emeran A Mayer ${ }^{3}$
}

${ }^{1}$ Department of Internal Medicine I, Gastroenterology, Hepatology \& Endocrinology, Medical University Innsbruck, Innsbruck, Austria

${ }^{2}$ WELBIO-Walloon Excellence in Life Sciences and

BIOtechnology, Louvain Drug Research Institute, Metabolism and Nutrition Research Group, Université catholique de Louvain, Brussels, Belgium ${ }^{3}$ Division of Digestive Diseases, G. Oppenheimer Center for Neurobiology of Stress and Resilience, David Geffen School of Medicine at UCLA, Los Angeles, California, USA

\section{Correspondence to} Professor Herbert Tilg, Department of Internal Medicine I, Medical University Innsbruck, Innsbruck 6020, Austria;

herbert.tilg@i-med.ac.at

Received 27 July 2016 Revised 13 September 2016 Accepted 16 September 2016 Published Online First 8 October 2016

\section{CrossMark}

To cite: Tilg $\mathrm{H}$, Cani PD, Mayer EA. Gut

2016;65:2035-2044.

\section{ABSTRACT}

The gut microbiota has recently evolved as a new important player in the pathophysiology of many intestinal and extraintestinal diseases. The liver is the organ which is in closest contact with the intestinal tract, and is exposed to a substantial amount of bacterial components and metabolites. Various liver disorders such as alcoholic liver disease, non-alcoholic liver disease and primary sclerosing cholangitis have been associated with an altered microbiome. This dysbiosis may influence the degree of hepatic steatosis, inflammation and fibrosis through multiple interactions with the host's immune system and other cell types. Whereas few results from clinical metagenomic studies in liver disease are available, evidence is accumulating that in liver cirrhosis an oral microbiome is overrepresented in the lower intestinal tract, potentially contributing to disease process and severity. A major role for the gut microbiota in liver disorders is also supported by the accumulating evidence that several complications of severe liver disease such as hepatic encephalopathy are efficiently treated by various prebiotics, probiotics and antibiotics. A better understanding of the gut microbiota and its components in liver diseases might provide a more complete picture of these complex disorders and also form the basis for novel therapies.

\section{INTRODUCTION}

Non-communicable diseases, also known as chronic diseases are still growing around the globe. According to WHO, the four main noncommunicable diseases are cardiovascular diseases, diabetes, cancer and chronic respiratory diseases. Strikingly, although our genetic background has not changed over the last century, the last three decades have been clearly associated with the globalisation of such diseases. The key triggering factors for many diseases associated with lifestyle include our changes in eating habits, exposure to specific xenobiotics or alcohol and tobacco consumption. Today, the role of the inhabitant of our gut, the so-called gut microbiota is also becoming recognised as a major environmental factor.

We knew from several decades that as human, we are composed of trillion cells, whereas the number of bacteria present in our gut and living in symbiotic harmony with our body is estimated to also reach such a comparable vast number of cells (eg, human body: around $3 \times 10^{13}$ eukaryotic cells; the microbiota around $4 \times 10^{13}$ colonising microbes). ${ }^{1}$

In utero, mammals are already exposed to microbiota or derived compounds, and this exposure expands rapidly after birth. ${ }^{2}{ }^{3}$ Therefore, it is suggested that in adults the gut microbiota composition and its activity may reflect in part the history of exposure to bacteria and environmental factors during early life. The divergences observed in composition of human gut microbiota are likely the result of multiple factors. For example, individual factors such as the genetic background, the age, the development of the immune system, the geographical location or any dietary modifications of short or longer durations may influence the gut microbiota composition. ${ }^{4-10}$

However, better understanding the correlations and eventually the potential causal link existing between the intestinal microbial community and the onset of metabolic disorders requires a comprehensive knowledge of the gut microbiota. Over decades, several methodological approaches have been used and are divided into two major categories: culture-dependent and culture-independent approaches. Culture-based techniques were the major methods used and are still used to analyse the microbiota composition. However, so far about only $30 \%$ of the gut microbes have been cultured. This does not mean that $70 \%$ of the gut microbiota are unculturable but simply suggests that it is very difficult to identify and to develop the optimal growth conditions for these organisms. This is likely due to the fact that the microbiota is a complex ecosystem, which is not easy to decode. Therefore, using culture-based methods alone is not suitable to provide a complete view of the resident microbes.

Although each technique has its own advantage or limitation, culture-independent methods have been useful to better illustrate the complexity of the microbiota composition. Thus, one may argue also that the choice for a specific approach depends on the key scientific questions that should be addressed. Among these methods, quantitative PCR (qPCR) and fluorescence in situ hybridisation have the advantage to be both highly sensitive, and eventually suitable to quantify specific bacterial groups. Conversely, using fingerprinting techniques allow a rapid comparison of profiles to identify the most abundant phylotypes (eg, within the same individual or between pathological situations), but, these methods require an a posteriori identification of specific bands by sequencing or hybridisation to identify known or unknown bacteria. More recently, phylogenetic microarrays have been developed and therefore used as a high-throughput technique. ${ }^{11}$ This method has contributed to progressively increase the knowledge of microbiota composition and is considered as a fast and semiquantitative method. ${ }^{12}$ However, the major caveat is that the detection of any species depends on the presence of known reference sequences on the array. As a consequence, this technique is not suitable for discovering novel taxa.

Over the last few years, next-generation sequencing methods have been developed and have dramatically increased the number of novel data 
and generated gigabases of sequences in one single run. The major advantage of these techniques is to allow to determine the relative abundance of both known and unknown bacteria. ${ }^{13}$ In most of the studies available, both culture-dependent and culture-independent methods have been used. Thus, thanks to the recent development of such analytical tools, the scientific community is progressively starting to highlight at least in part the composition of the human intestinal microbiota. ${ }^{14}$ For example, different catalogues of up to 10 million non-redundant genes have been published ${ }^{15} 16$ and novel taxa have recently been identified or associated with diseases progression. ${ }^{17-20}$

In general, it is considered that a healthy microbiota-host symbiosis is resulting from a stable GI tract environment, rich in nutrients and providing mutualistic interests. The microbiota will ensure to provide essential nutrients (eg, production of vitamins, increase bioavailability of essential nutrient) by assisting digestion of non-digestible compounds (eg, dietary fibres), thereby allowing the growth of a metabolically active population of bacteria. It is commonly accepted that any perturbations of the relationship existing between host and microbes may be associated with the pathogenesis of several major diseases, thereby highlighting the role of microbes outside the intestinal tract. However, while important progress has been achieved thanks to metagenomics analyses, and association between specific taxa and diseases, a vast number of key questions remain unanswered, including the demonstration of a causal link in the onset of non-communicable diseases such as obesity, diabetes and cardiometabolic diseases. Nevertheless, it is now widely accepted that the gut microbiota composition and its metabolic capacity contribute to regulate host metabolism. We will herein limit our discussion on a key organ which is at the interface of many interactions between the gut and host metabolism, that is, the liver.

\section{NON-ALCOHOLIC FATTY LIVER DISEASE, METABOLIC INFLAMMATION AND MICROBIOTA}

The liver is structurally and functionally heterogeneous and contains a large number of different cell types, such as hepatocytes, immune cells (innate and adaptive immune system) including Kupffer cells, lymphocytes, but also stellate cells and progenitors.

The liver is at the crossroad between the portal blood flow coming from the intestinal circulation and peripheral organs. This anatomical position offers to the liver specific interactions with many compounds coming from the digestion of nutrients transiting throughout the gut and also with other microbiotaderived signals. In physiological situations, nutrients and bacterial compounds are delivered to the liver via the portal circulation and contribute to the host homeostasis. Thus, the major border between the intestinal lumen (ie, microbes, digested food, xenobiotic), the portal blood and the liver is the gut barrier. This barrier comprises the intestinal mucosa of the host and also several other highly complex and dynamic cells and structures that are at the interface between the microbiota, the liver and other organs.

Although the gut lining acts as an active barrier against the translocation of bacteria and/or microbial-derived products, a small quantity of these compounds may enter the portal venous blood. Thus, a fine-tuned sensitive balance takes place to limit excessive responses between an expected protective immune response against exogenous insults and immune tolerance.

The pathogenesis of different liver diseases has been associated with changes in the gut microbiota composition. Among these, non-alcoholic fatty liver disease (NAFLD) is a common clinical syndrome, induced by factors other than alcohol consumption or any other well-established liver injury. NAFLD is pathologically characterised by a diffuse accumulation of fat in the liver cells (steatosis) and is also a key factor involved in the development of insulin resistance, type 2 diabetes and cardiovascular risk. Moreover, a certain proportion of patients with NAFLD will evolve into non-alcoholic steatohepatitis (NASH) and eventually cirrhosis and hepatocellular carcinoma. $^{21}$

\section{Gut microbes and hepatic steatosis: mechanisms}

Studies have revealed that the alteration of gut microbiota may play an important role in the development and progression of NAFLD. For example, more than 20 years ago, seminal papers have shown that changing the microbiota composition by using prebiotics such as inulin-type fructans reduces hepatic steatosis and de novo lipogenesis. ${ }^{22-25}$ These studies were the first showing a decrease in plasma triglycerides and very low-density lipoprotein production in prebiotic-fed rats. The triglycerides-lowering action of prebiotics is mediated by the inhibition of all lipogenic enzymes, namely acetyl-coenzyme A carboxylase (ACC), fatty acid synthase (FAS), malic enzyme, ATP citrate lyase and glucose-6-phosphate dehydrogenase (figure 1). ${ }^{26}$ In 2003, Letexier et $a l^{27}$ showed that changing the microbiota by using prebiotic decreased hepatic lipogenesis and plasma triglycerides, thereby showing one of the first metabolic links between microbiota and liver in humans. The fermentation of prebiotics by gut microbes increases the abundance of short chain fatty acids in the caecum and also in the portal vein blood, where the concentration of both acetate and propionate

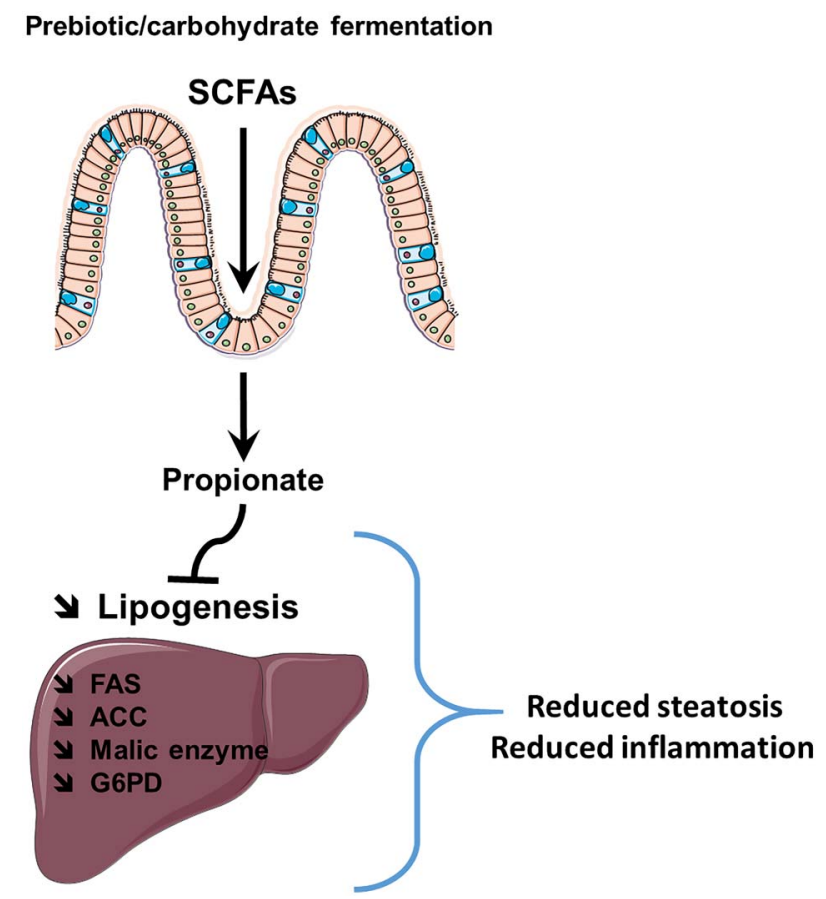

Figure 1 Prebiotic fermentation reduces hepatic steatosis. Gut microbiota is using prebiotic and fermentable carbohydrates as energy sources; hence, short chain fatty acids (SCFA) production such as propionate may cross the gut barrier and reach the liver through the portal vein blood. Propionate inhibits lipogenesis by acting on the transcription of several rate-limiting step enzymes involved in de novo lipogenesis. As a consequence, these fibres and their metabolites are putative tools to reduce steatosis and inflammation. ACC, acetyl-coenzyme A carboxylase; FAS, fatty acid synthase; G6PD, glucose-6-phosphate dehydrogenase. 
is doubled. ${ }^{28-30}$ Data suggest that propionate may contribute to reduce hepatic lipogenesis, whereas acetate is a lipogenic substrate (figure 1). ${ }^{31} 32$

In 2005, Daubioul et $a l^{33}$ showed in a pilot study with seven patients with NASH (confirmed by biopsy) that after 8 weeks, prebiotic feeding decreased significantly hepatic inflammatory markers. More recently, 66 patients with NASH were randomly divided into two groups. The first group receiving a symbiotic (ie, mix of Bifidobacterium longum and prebiotics (inulin-type fructans) combined with lifestyle modification, whereas the second group received a placebo with lifestyle modifications. ${ }^{34}$ After 24 weeks of treatment, the authors found that the modulation of the gut microbiota significantly reduced inflammatory markers (ie, tumour necrosis factor- $\alpha, C$ reactive protein) as well as steatosis and NASH activity index. Altogether, these data strongly suggest that the metabolic activity of microbes may contribute to the regulation of hepatic lipogenesis and eventually the development of steatosis. But, it is worth noting that a causal impact of specific bacteria or metabolites was not shown in these studies.

However, this causality between weight gain, fat mass and steatosis has been shown by Bäckhed et al, ${ }^{35}$ who discovered that mice raised in the absence of microorganisms (axenic or germ free) exhibited a lower total body fat mass than mice harbouring a normal gut microbiota. They also found that to conventionalise germ-free mice with a gut microbiota from normal mice increases body fat content, insulin resistance and liver fat. ${ }^{35}$ The authors observed that colonising the gut with microbiota was accompanied by a twofold increase in hepatic triglyceride and a higher hepatic de novo lipogenesis measured by the expression of several key factors and enzymes such as hepatic carbohydrate responsive element binding protein, sterol responsive element binding protein 1, ACC and FAS. ${ }^{35}$ Among the mechanisms proposed, the authors found that conventionalised mice had an increased intestinal saccharides absorption and delivery of glucose to the liver that eventually participates in higher lipogenesis (figure 1).

Another piece of evidence supporting a role for the gut microbiota in the development of NAFLD has been demonstrated by Le Roy et al. They observed that upon a high-fat diet treatment, a certain proportion of mice develop obesity, insulin resistance and NAFLD. Strikingly, in such set of rodent experiments, the genetic background is exactly the same as well as the dietary environment; however, for different unknown reasons the microbiota may respond differently to diet. Despite a majority of the mice investigated do develop metabolic disorders (ie, higher glycaemia, systemic inflammation and steatosis), some other were resistant to the diet-induced metabolic disorders and were considered as 'non-responders' versus 'responders'. Interestingly, the microbiota composition of 'non-responders' was different from 'responders'. By using germ-free mice, they demonstrated that the susceptibility to develop NAFLD was transmissible by the gut microbiota transplantation. Thus, this set of experiments clearly shows that specific bacterial species are associated with the onset of NAFLD upon a dietary trigger such as high-fat feeding. ${ }^{36}$

Dysbiosis of the gut microbiota has been associated with NAFLD in mice and in humans. ${ }^{37} \mathrm{~A}$ recent review by Abdou et $a l^{38}$ discusses multiple studies performed in humans showing that dysbiosis is accompanied by an abnormal shift of the gut microbiota as compared with healthy individuals. However, there is still no clear consistency or specificity towards key gut microbes to explain predisposition to develop NAFLD.

Although this is not the scope of this review, evidence suggests that obesity and insulin resistance are the key risk factors for development of NAFLD. Numerous recent studies have shown that the gut microbiota composition and its activity are different during obesity and type 2 diabetes. ${ }^{5}$ 39-42 Although, it is still too early to link these metabolic situations with the systematic presence or the absence of specific taxa, there is a consensus on the fact that during obesity and diabetes, the bacterial diversity is lower than in healthy subjects. Recently, Le Chatelier et al identified that the number of bacterial genes (ie, microbial gene richness) follows a bimodal distribution of microbial genes. This led the authors to propose a stratification of subjects on either harbouring 'low gene count' (LGC) or 'high gene count' (HGC) according to the number of genes present in their microbiome. ${ }^{40}$ Interestingly, specific taxa have been linked with both the metabolic situations and the gene counts. For example, the authors proposed that butyrate producers such as Anaerotruncus colihominis, Butyrivibrio crossotus, Faecalibacterium prausnitzii and Roseburia inulinivorans were less abundant in patients with LGC and may be associated with an obese phenotype. In addition, other specific beneficial microbes such as Bifidobacterium spp and Akkermansia muciniphila were also less abundant. Conversely, increased abundance of Ruminococcus gnavus, Ruminococcus torques, Campylobacter and Shigella was observed. ${ }^{40}$ A recent study has found an association between the abundance of Prevotella copri, Bacteroides vulgatus and insulin resistance in humans. They also described that P. copri aggravates insulin resistance and triggers glucose intolerance in rodents, thereby showing a putative causal link. ${ }^{43}$ Interestingly, Dao et al found in obese humans, the abundance of A. muciniphila was inversely related to visceral fat mass, fasting glycaemia and adipocyte size. They also observed that subjects categorised as HGC and higher A. muciniphila abundance exhibited improved insulin sensitivity markers and other cardiometabolic risk factors. ${ }^{44}$ It is worth noting that this bacterium has been shown to protect against diet-induced obesity, reinforce the gut barrier and reduce low-grade inflammation in rodents. ${ }^{18}$ Therefore, although the exact implication of the taxa mentioned earlier in the onset of metabolic disorders remains to be proven in humans, several studies are now consistently showing such kind of associations.

\section{From the gut to the liver: impact of inflammation}

Gut bacteria are able to interact with host cells via specific molecules called pattern recognition receptors (PRRs). These PRRs will recognise particular molecular patterns of bacteria and other microorganisms, namely the pathogen-associated molecular patterns (PAMPs). Among the different PRRs, toll-like receptors (TLRs) are the most studied. In 2007, Cani et al discovered that gut microbes were involved in the onset of insulin resistance, low-grade inflammation and diabetes, by activating TLR signalling pathways. They found that constituents of Gram-negative bacteria (ie, lipopolysaccharides (LPS)), which are circulating at a very low level in the blood, were able to trigger low-grade inflammation and altered glucose metabolism. $^{45}$ This phenomenon was called metabolic endotoxemia (figure 2).

Besides the apparent inflammatory tone and insulin resistance, they found that metabolic endotoxemia also triggers liver fat accumulation. ${ }^{45}$ This effect was abolished in mice lacking the LPS receptor complex CD14/TLR4, ${ }^{45} 46$ thus showing a direct link between gut microbial compounds and the development of hepatic steatosis. Along this line, as described earlier in this review, changing the microbiota by using prebiotics reduces hepatic steatosis and both hepatic and systemic inflammation in both rodents and humans (figure 1). ${ }^{33} 3447$ 


\section{Dysbiosis \\ HFD, Alcohol, Low fibres}
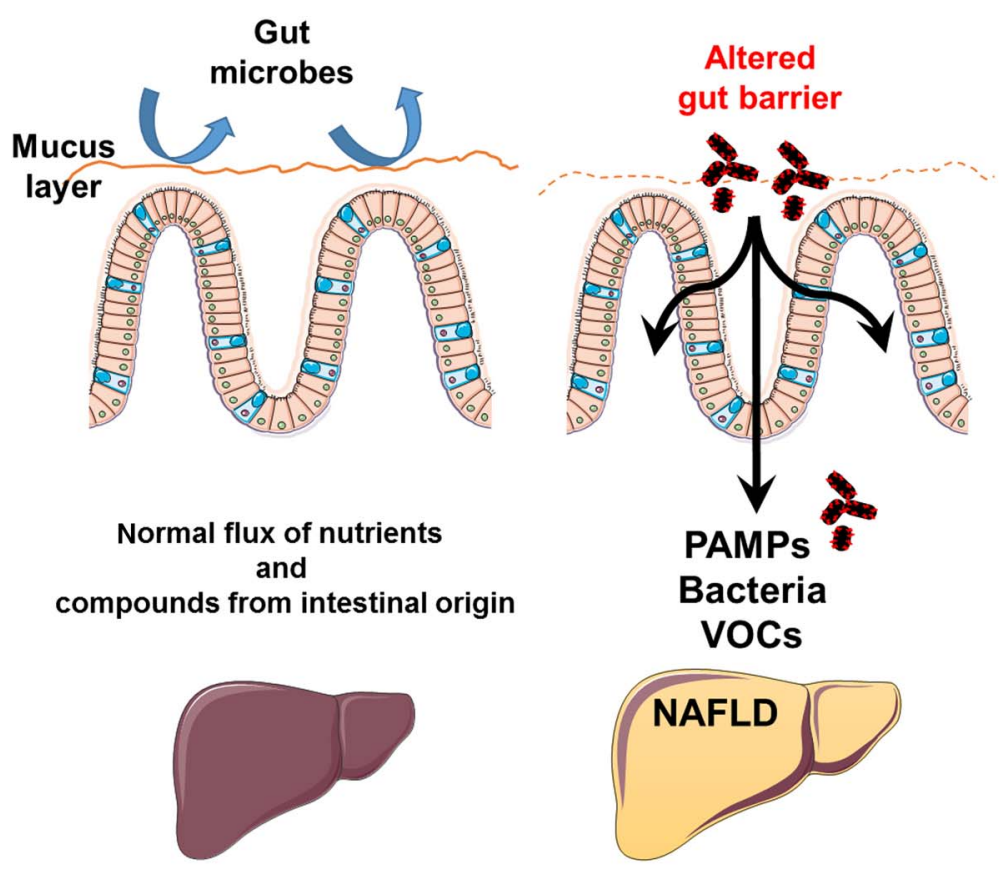

Dysbiosis
HFD, Alcohol, Low fibres
X-factor

Altered

gut barrier
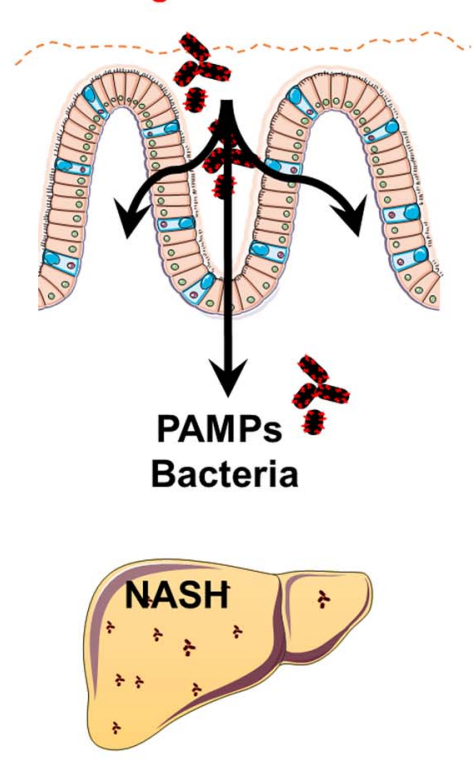

Alteration of bioactive lipids production ?

Inflammasomes? Innate immune response? Cyp450?

Figure 2 Disease progression model from normal liver to non-alcoholic fatty liver disease (NAFLD) and non-alcoholic steatohepatitis (NASH): contribution of the gut-liver axis. The gut lining is composed of several actors (eg, mucus layer, symbiotic gut microbes) contributing to maintain the gut barrier. Changes in dietary habits such as reducing the ingestion of fibres together with higher consumption of saturated fat, alcohol and specific xenobiotics may contribute to change the microbiota composition and to alter the gut barrier function. The subsequent leaky gut is associated with the translocation of bacteria or fragments of bacteria that trigger the accumulation of liver fat and inflammation. Both NAFLD and NASH are associated with specific changes at the level of the immune response, as well as endogenous production of bioactive lipids. This dysbiosis is also associated with the production of potential organic compounds reaching the liver and changing its metabolic activity. Altogether, several mechanisms are involved in the onset of NAFLD and NASH, most of them being characterised by a central role of the gut microbiota. HFD, high-fat diet; PAMP, pathogen-associated molecular pattern; VOC, volatile organic compound.

Inflammasomes are considered as key sensors for endogenous and exogenous PAMPs or damage-associated molecular patterns (DAMPs). They control cleavage of pro-inflammatory cytokines such as pro-interleukin (IL)-1 $\beta$ and pro-IL-18. In 2012, Henao-Mejia et al showed that genetic inflammasomes deficiency induced a change in the gut microbiota composition. They illustrated that the genetically induced dysbiosis led to abnormal accumulation of bacterial products in the portal circulation and consequently increased hepatic steatosis. ${ }^{48}$ The role of inflammasomes in the onset of NAFLD has been recently reviewed (figure 2). ${ }^{49}$

More recently, Duparc et al investigated the impact of hepatocyte-specific deletion of myeloid differentiation primary response gene 88 (MyD88), a central adaptor molecule for the majority of these TLRs (with the exception of TLR3 and certain TLR4 signals), and members of the inflammasomes (IL-1 receptor and IL-18 receptor). They found that in absence of MyD88 specifically in the hepatocyte, mice were predisposed to glucose intolerance, inflammation, liver fat accumulation and hepatic insulin resistance, and this was independent of body weight and adiposity. ${ }^{50}$ In addition, this deletion profoundly affected the gut microbiota composition, therefore, showing that host genetics also contribute to shape the microbiota and in turn host metabolism. Among the potential mechanisms, they found that hepatocyte MyD88 is involved in the control of the synthesis of bile acids and several bioactive lipids, all involved in the regulation of glucose, lipid metabolism and inflammation. ${ }^{50}$

Interestingly, Duparc et al found that in human obese subjects who developed NASH, different factors involved in the production of bioactive lipids synthesis (ie, different cytochromes P450) were decreased, they also linked several transcriptional factors with the switch from NAFLD to NASH. However, the microbiota of these patients was not studied. ${ }^{50}$

Together, this suggests the existence of a crosstalk between microbes and host liver cells that control metabolism. In parallel to PAMPs, DAMPs and bioactive lipids, recent data suggest that volatile organic compounds (VOCs) produced by the microbial metabolism may differentiate obese patients with NAFLD from healthy individuals (figure 2). ${ }^{51}$ It is well known that the absorption of VOCs such as ethanol and other toxic molecules from the gut may be deleterious for the liver. However, the recent discovery that more than 40 gut-derived VOCs are present in the portal vein of mice developing NAFLD strongly suggests that besides the gut microbiota composition the metabolic activity of bacteria may expose the liver to xenobiotic compounds (figure 2, box 1 ). 
Box 1 Microbiota and non-alcoholic fatty liver disease (NAFLD)

- Gut microbiota contributes to the regulation of de novo hepatic lipogenesis

- Specific nutrients such as fat and alcohol change microbiota composition in a harmful manner, whereas prebiotics counteract these effects

- Both innate immune system and xenobiotic metabolism control liver lipid metabolism via mechanisms involving bacterial components and metabolites

- Hepatic innate immunity controls liver bioactive lipids production and contribute to switch from NAFLD to non-alcoholic steatohepatitis.

\section{ALCOHOLIC LIVER DISEASE AND MICROBIOTA}

The diverse clinical picture of alcoholic liver disease (ALD) might be caused by various confounders including genetics, immunity, dietary components and the gut microbiota. ${ }^{52} \mathrm{An}$ altered microbiota or commonly phrased 'dysbiosis', an impaired intestinal barrier and endotoxemia are well-known features of advanced ALD. ${ }^{53}$ Mutlu et $a l^{54}$ studied the mucosa-associated colonic microbiome in alcoholics with and without ALD and in healthy subjects. A subgroup of patients exhibited a dysbiosis with lower median abundances of Bacteroidetes and higher ones of Proteobacteria. Interestingly, changes corrrelated with endotoxemia. Intestinal permeability and dysbiosis, however, might also play a role in other alcoholrelated aspects such as alcohol-dependence severity. ${ }^{55}$ This study suggested that indeed a gut-brain axis might exist in alcoholism providing certain evidence that the gut microbiota regulates behavioural disorders such as alcohol dependence. Therefore, it has been increasingly recognised that the gut microbiota might be relevant in alcohol-related human disorders.

The microbiota might be of exceptional relevance in severe alcoholic hepatitis (AH). ${ }^{56}$ Llopis et $a l^{56}$ showed that disease severity of human $\mathrm{AH}$ can be transferred via faecal microbial transplantation (FMT) both to germ-free (GF) and conventionalised mice. First, the authors characterised the gut microbiota of various groups of patients with $\mathrm{AH}$ describing that $\mathrm{AH}$-related dysbiosis was characterised by an increase in Bifidobacteria, Streptococci and Enterobacteria, whereas certain species such as Clostridium leptum or F. prausnitzii, both well-established antiinflammatory strains, were decreased. ${ }^{17}$ These protective strains were also negatively correlated with certain clinical parameters such as bilirubin levels. In FMT experiments, where stool from patients with or without $\mathrm{AH}$ was used, the authors found that severity of liver disease in animals was dramatically aggravated after performing FMT from a patient with severe AH. In contrast, if for FMT a human donor without severe $\mathrm{AH}$ was used, disease phenotype was mild. Importantly, disease could also be transferred to conventional mice and a second subsequent FMT from an alcoholic patient without $\mathrm{AH}$ even improved liver disease after having received FMT from a donor with severe AH before. This important study delivers some key messages to this rapidly evolving and exciting field: (i) there is a clear microbiota signature in severe $\mathrm{AH}$, (ii) disease can be transferred from man to mouse and (iii) thereby suggesting that certain 'pathobionts' might indeed exist. It seems now crucial to enforce further studies trying to identify members of the microbiota, which are drivers of this often fatal disease in order to define new targets for better therapies.

Whereas as discussed certain members of the microbiota might drive ALD (ie, pathobionts), other members might exert beneficial effects in the disease process and act protective. This fits with recently presented data where Chen et $a l^{57}$ showed that ALD is aggravated in germ-free mice and in these studies hepatic ethyl alcohol $(\mathrm{EtOH})$ metabolism was enhanced and susceptibility to binge-like alcohol drinking increased. Another exciting research area deals with the key questions how an intact intestinal microbiota is regulated. Local intestinal immunity including defensins and other antimicrobial factors might constitute major players in the regulation of the microbiota. ${ }^{58}$ Chronic alcohol consumption decreases intestinal levels of antimicrobial-regenerating islet-derived (REG)3 lectins. Intestinal-specific deficiency in REG3B or REG3G increased numbers of intestinal bacteria and their translocation to mesenteric lymph nodes and the liver aggravating ethanol-induced steatohepatitis. In contrast, overexpression of REG3G in the intestinal epithelium protected animals from ethanol-induced liver injury (figure 2). This overexpression was directly associated with reduced bacterial colonisation in the intestinal mucosa and reduced bacterial translocation. This study highlights besides the potential contribution of the gut microbiota to disease pathogenesis the importance of intestinal immunity.

Diet might reflect another major confounder in ALD, with the potential to either improve or aggravate underlying disease. Indeed, an unsaturated fat diet (corn oil enriched) exacerbated ethanol-induced endotoxemia and worsened liver disease in contrast to a saturated diet enriched in medium chain triglycerides. ${ }^{59}$ An unsaturated diet was accompanied by additonal alterations in the gut microbiota with reduction in Bacteroidetes and an increase in Proteobacteria and Actinobacteria. This study highlights the importance of dietary factors in alcohol-related liver disease potentially by manipulating the gut microbiota. To conclude, the gut microbiota has evolved as major player in ALD. Alcohol disrupts the intestinal microbiome, alters the intestinal barrier and might affect various other intestinal functions such as mucosal immunity. Therapies such as various diets, prebiotics and probiotics might have the potential to influence and correct these disturbances and appear as potential future therapies for these diseases. ${ }^{60}$ In addition, manipulation of the gut microbiota by prebiotics/probiotics or FMT selectively targeting key pathobionts might reflect another promising treatment strategy. Treatment of ALD and AH has entered a new exciting area (box 2).

\section{PRIMARY SCLEROSING CHOLANGITIS AND MICROBIOTA}

Primary sclerosing cholangitis (PSC) is commonly observed in patients with IBD and recent studies have investigated the gut microbiome in this disorder. ${ }^{61}$ Here, $16 \mathrm{~S}$ rRNA gene sequencing performed in 85 patients with PSC demonstrated that such patients independent of the presence of IBD had a reduced bacterial diversity compared with healthy controls. Interestingly, approximately 11 genera were reduced in PSC, whereas Veilonella genus showed an increase. In summary, this first PSC study observed a clear gut signature in PSC distinct from healthy controls and patients with UC without liver disease suggesting that the intestinal microbiota (IM) could reflect a relevant player in PSC. These findings are strengthened by another recently published study where authors also observed an increase in Veilonella species. ${ }^{62}$ Veilonella has been associated with other chronic inflammatory disorders including fibrotic conditions and liver cirrhosis where subjects have higher colonic 


\section{Box 2 Microbiota and alcoholic liver disease}

- Human alcoholic liver disease is characterised by a profound dysbiosis

- An impaired intestinal epithelial barrier is an early event in many experimental models of alcoholic liver injury even preceding intestinal dysbiosis/altered microbiome

- Impaired GI barrier might result in rapid endotoxemia

- An altered intestinal microbiome is observed in various experimental liver disease models and microbiome changes might be disease-specific

- Preclinical and clinical studies suggest an important role for the gut microbiota in alcohol-related disorders

Veilonella levels. ${ }^{63}$ Another smaller study investigated mucosa-associated microbiota in 20 patients with PSC, 19 with accompanying IBD. ${ }^{64}$ Investigators observed an increase in mucosal Barnesiellaceae at the family level and Blautia at the genus level. Germ-free, multidrug resistance 2 knockout mice $\left(\mathrm{mdr} 2^{-/-}\right.$mice) show exacerbated biochemical and histological features of PSC and increased cholangiocyte senescence further supporting the notion that gut microbiota might play a role in disease process. ${ }^{65}$ Interestingly, primary bile acids were similar, whereas secondary bile acids were absent in germ-free $\mathrm{mdr}^{-/-}$ mice. This study also implicates an important role for the gut microbiota and certain metabolites such as secondary bile acids in the protection against biliary injury. PSC and microbiome research is a rapidly emerging topic and one of the most challenging issues will remain to understand and identify differences in the microbiome of patients with PSC with and without IBD (box 3).

\section{LIVER CIRRHOSIS AND MICROBIOTA \\ Liver regeneration}

Besides many immune factors especially various cytokines such as IL-6, the gut microbiota might also affect and regulate liver regeneration. After antibiotic therapy, especially when using ampicillin, the number of CD1d-dependent natural killer T (NKT) cells was markedly reduced after partial hepatectomy. These NKT cells and activated Kupffer cells produced high amounts of the cytokines interferon- $\gamma$ and IL-12 and neutralisation especially of IL-12 abrogated the negative effects of antibiotic therapy on liver regeneration. Antibiotic therapy after hepatectomy therefore could have a negative effect on liver regeneration potentially also in humans. ${ }^{66}$ Indeed, a recent study suggests that specific intestinal bacteria are closely associated with expression of certain

\section{Box 3 Microbiota and primary sclerosing cholangitis}

- Specific microbiome signature is found in primary sclerosing cholangitis

- Veilonella is increased in human primary sclerosing cholangitis and other human chronic inflammatory disorders

- Primary sclerosing cholangitis microbiome signature is different than the one observed in patients with UC without liver disease

- Multidrug resistance 2-deficient germ-free mice exhibit more pronounced primary sclerosing cholangitis-like liver disease genes in a regenerating liver. ${ }^{67}$ Partial hepatectomy resulted in an upregulation of more than 6000 bacterial microbiotal genes, some of them involved in bile acid metabolism and hepatocyte proliferation. This type of surgery was accompanied by massive changes in the IM, for example, an increase of Bacteroidetes and Rikenellaceae and a decrease in Clostridiales, Lachnospiraceae and Ruminococcaceae. It is well established that conventional inflammatory cytokines and Wnt factors control tissue repair and liver regeneration. ${ }^{68}$ Although sterile inflammation may play here the dominant role, it may well be that the gut microbiota acts as another driving force of this type of inflammation. Therefore, the gut microbiota seems to be a so far ignored player for successful liver regeneration.

\section{Liver cirrhosis}

Patients with liver cirrhosis show an increase in the translocation of intestinal bacteria and increased circulating concentrations of bacterial DNA suggesting that indeed the GI tract might reflect the major source of pathogens/pathobionts. Bacterial translocation plays a key role in the appearance of systemic infections in liver disorders and gut bacteria might differ in their ability to translocate or bypass the intestinal barrier. In gnotobiotic mice, enteric bacteria such as Escherichia coli, Proteus and Enterobacter are associated with a higher incidence of bacteraemia, because these bacteria translocate more efficiently from the GI tract than anaerobes. The faecal microbial community in patients with liver cirrhosis in comparison with healthy individuals had been characterised. ${ }^{69}$ In this study, the faecal microbial communities were analysed by 454 pyrosequencing of the $16 \mathrm{~S}$ ribosomal RNA V3 region and by subsequent real-time qPCR. Bacteroidetes was significantly reduced, whereas Proteobacteria and Fusobacteria were highly increased in the cirrhosis group. Importantly, the prevalence of potentially pathogenic bacteria, such as Enterobacteriaceae and Streptococcaceae, with decreased presence of beneficial populations such as Lachnospiraceae might affect clinical phenotype and even prognosis in these patients. An important study recently assessed the gut microbiome in relation to cirrhosis severity, its stability over time and alterations with decompensation. ${ }^{70}$ Here, the authors used multitagged pyrosequencing and observed that progressive changes in the gut microbiome accompany cirrhosis and become more severe in case of decompensation. The ratio of autochthonous to nonautochthonous taxa was calculated as the cirrhosis dysbiosis ratio (CDR) and a low number indicated dysbiosis. CDR was highest in controls, lower in compensated and lowest in decompensated and inpatients and negatively correlated with endotoxin plasma levels. Importantly, in their study microbiota was significantly different between infected and non-infected cirrhotics. These authors recently showed that patients with liver cirrhosis exhibit a profound salivary dysbiosis. ${ }^{71}$ In this study, in 102 studied patients stool and saliva microbiome differed markedly on principal component analysis. The salivary microbiome with previously diagnosed hepatic encephalopathy (HE) showed an increase in pathogenic Enterobacteriacceae and Enterococcaceae. Changes in stool microbiota, however, correlated better with signs of systemic inflammation in these patients. The authors concluded that patients with liver cirrhosis exhibited both salivary and stool dysbiosis and changes were more pronounced in patients requiring the follow-up hospitalisations.

Qin et al investigated 98 patients with liver cirrhosis and 83 healthy controls using metagenomic analysis. ${ }^{72}$ They showed a profound dysbiosis and interestingly 54\% of the patientenriched taxonomically assigned species were of buccal origin, suggesting that the oral microbiota when present in the lower 
GI tract could contribute to disease process/severity. All these studies clearly support a major role for the intestinal microbiota in liver cirrhosis. At least in animal models the gut microbiota changes significantly in the progression of liver disease. ${ }^{73}$ Here, the authors induced liver disease by a streptozotocin high-fat diet leading to steatosis, fibrosis and finally hepatocellular carcinoma. Several bacterial species such as various Bacteroides, Atopobium spp, Clostridium cocleatum, Clostridium xylanolyticum and Desulfovibrio increased over disease progression and correlated with endotoxemia and clinical features. Liver cirrhosis can be considered as a prototypic microbiota-driven disorder (box 4).

\section{GUT-BRAIN AXIS AND MICROBIOTA IN HE}

A series of preclinical and some clinical studies have clearly established the bidirectional communication between the brain and the gut microbiota. ${ }^{74-76}$ Several communication channels by which gut microbes can signal to the brain have been identified, including neuronal, endocrine and immune-mediated pathways. ${ }^{77}$ The type and amount of gut microbial information that reaches the brain is greatly dependent on the regional intestinal milieu the microbes inhabit (influenced by regional intestinal transit, mucus secretion, production of antimicrobial peptides and intraluminal release of neuroactive substances such as noradrenaline), permeability of the gut epithelial barrier and the blood-brain barrier and the clearance of gut microbial metabolites through the liver, all of which are highly variable. ${ }^{74} 78$ Altered gut microbiome brain signalling, including the development of low-grade diet-induced 'metabolic endotoxemia' has been implicated in the pathophysiology of several psychiatric and neurological disorders, ${ }^{75} 79$ as well as in the pathophysiology of HE. ${ }^{80} \mathrm{HE}$ is a severe neuropsychiatric complication of both acute and chronic liver failure. It is characterised by deficits in psychiatric, neurocognitive and motor functions, ranging from minimal HE including reversal of sleep-wake cycle, shortterm memory loss, poor concentration and deficits that may only become apparent on formal neurocognitive testing to the most severe symptoms of delirium and coma. ${ }^{81-83}$

For more than 100 years, increased ammonia levels and their toxic effects on the brain have remained the most widely accepted mechanism underlying the development of $\mathrm{HE},{ }^{84}$ and various ammonia-lowering strategies (lowering of protein ingestion, broad-spectrum antibiotics, lactulose) remain the standard of care for HE today. Ammonia has long been thought to be produced primarily by urease producing gut microbiota (figure 3), but more recent evidence implicates an important role of the viscera (in particular small intestine and kidneys) as well. ${ }^{85}{ }^{86} \mathrm{In}$ addition to such increased generation of ammonia, the compromised

\section{Box 4 Microbiota and liver cirrhosis}

- Infections remain the most common cause of death in these patients

- Liver cirrhosis is associated with a profound dysbiosis; Bacteroidetes are significantly reduced, whereas Proteobacteria and Fusobacteria are highly increased in the cirrhosis group

- Oral microbiota becomes highly present in the intestine

- Advanced liver diseases and subsequent decompensation of liver cirrhosis is characterised by worsening of dysbiosis

- Typical complications of advanced liver disease such as hepatic encephalopathy are substantially microbiota-driven

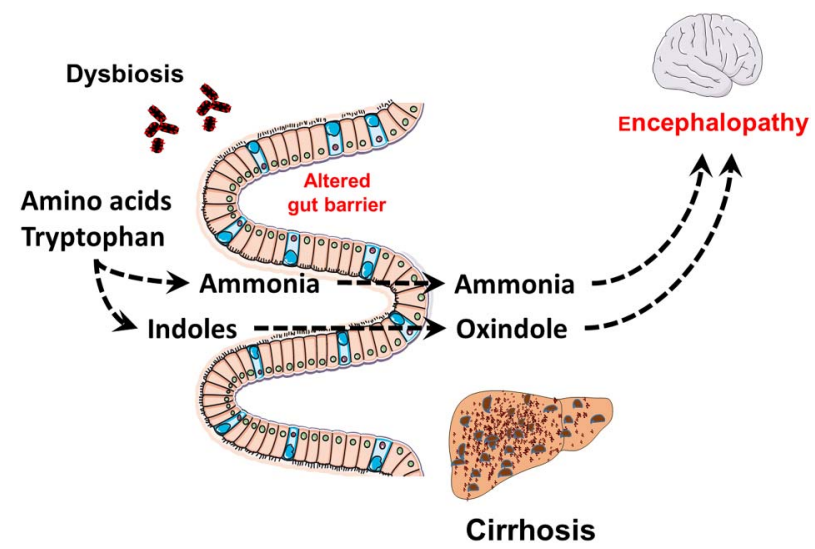

Figure 3 Gut-liver-brain axis and contribution to encephalopathy. Gut bacteria metabolise amino acids into specific metabolites including indoles and ammonia. Besides the altered gut barrier function, cirrhosis is also characterised by an alteration of the detoxification of ammonia, directly coming from the gut, as well as other specific compounds or derivatives such as oxindole that are by-products of indoles. Thus, among the different mechanisms linking metabolites of microbes and encephalopathy, several strong associations have been found between both ammonia and indoles/oxindole levels.

clearance of portal venous ammonia by the cirrhotic liver and increased uptake of ammonia by astrocytes in the brain are thought to play important roles in development of neurological symptoms (figure 3). The original ammonia hypothesis has been expanded through the identification of additional synergistic factors including regional increases in GABAergic tone in certain brain regions, ${ }^{87-89}$ compromised intestinal barrier function, lowgrade systemic inflammation, neuroinflammation ${ }^{80}$ and alterations in the gut microbiome..$^{70}$ It is likely that many of these factors interact resulting in altered brain function. For example, compromised barrier function both at the gut and brain level can increase the amount of inflammatory and neuroactive metabolites that reach the brain. It is conceivable that under these conditions, GABA generated by certain gut microbes ${ }^{90}$ could reach the brain and contribute to the increased GABAergic tone observed in HE. ${ }^{87}$ Gut-generated inflammatory signals can reach the brain via different mechanisms, including the activation of intestinal and vagal afferent pathways, direct cytokine and LPS signalling through the bloodstream, facilitated by increased permeabilities of intestinal and blood-brain barriers and migration of primed monocytes through the blood-brain barrier (figure 3). ${ }^{80}$ Neuroinflammation results from regional activation of glial cells, the resident macrophages that make up the majority of cells in the brain, which upon stimulation generate a series of cytokines with effects on neuronal function and glutaminergic neurotransmission.

Direct evidence for the important role of the gut microbiota in the development of brain changes and cognitive dysfunction in HE comes from recent clinical and preclinical studies. ${ }^{91}{ }^{92}$ In a comprehensive study in 147 cirrhotics and 40 healthy controls, Ahluwalia et al performed systemic inflammatory assessment, cognitive testing, assessment of regional brain astrocyte and metabolite changes by MR spectroscopy (MRS) and assessment of neuronal integrity and oedema by diffusion tensor imaging, 16S RNA sequencing of microbial composition and inference of the metagenomic content of samples from $16 \mathrm{~S}$ data. ${ }^{83}$ As expected, patients with HE had greater impairment of cognitive function, more evidence for systemic inflammation, dysbiosis and hyperammonaemia compared with healthy controls and cirrhotics 
without HE. Cirrhotics with HE had a higher relative abundance of autochthonous taxa and a higher abundance of Staphylococcaceae, Enterococcaceae, Porphyromonadaceae and Lactobacillaceae compared with cirrhotics without HE and with healthy controls. Imputed stool microbial functionality in cirrhotics with HE was related to functions of endotoxin, endotoxinprotein synthesis and a shift towards an ammonia generating amino acid profile (figure 3). Intriguingly, specific microbial taxa (autochtonuous taxa negatively, Enterobacteriaceae positively) differentially correlated with hyperammonia-associated astrocytic changes on MRS (increased glutamate/glutamine ratios, reduced myoinositol). On the other hand, the taxa Porphyromonadaceae were only correlated with neuronal changes as seen on DTI of white matter integrity, but not with ammonia levels. These findings for the first time demonstrate correlations between altered gut microbial taxa and specific neuronal and astrocytic changes in the brain of cirrhotics with HE. Kang $e t a l^{92}$ further characterised the relative role of the gut microbiota and ammonia levels in the development of neuroinflammation in a $\mathrm{CCl}_{4}$-induced model of cirrhosis in GF and in conventional mice. When compared with non-cirrhotic GF animals, cirrhotic GF mice showed higher ammonia levels, but no evidence of systemic or brain-related inflammatory changes. In contrast, conventional cirrhotic mice had intestinal dysbiosis as well as evidence for inflammatory brain changes. Similar correlations between gut microbial taxa, inflammatory marker and ammonia were identified in the earlier human study. Besides the role of ammonia, other data have shown that other products resulting from the metabolisation of amino acids such as tryptophan can also lead to the production of compounds such as indoles that can be further transformed into oxindole. For example, Riggio et al $l^{93}$ showed that blood levels of total indole compounds were higher in patients with HE (figure 3). They also found that oxindole levels were further higher in patients with liver cirrhosis. Interestingly, both ammonia and oxindole levels are positively correlated, thereby suggesting a similar gut-liver-brain axis in the physiopathology of HE. Another study by Montagnese $e t a l^{94}$ also showed a strong association between ammonia and indole/oxindole levels and the alteration of EEG, a sign of neurophysiological abnormalities that can already be observed in more compensated patients (figure 3).

In summary, extensive preclinical and clinical evidence supports a crucial role of altered communication between the gut, its microbiota and the brain in the development of HE. A series of reported findings, including small intestinal overgrowth, gut dysbiosis, increased gut permeability, metabolic endotoxemia and brain changes (including regional neuroinflammation and possibly increased GABAergic tone) are consistent with this concept. These findings have important therapeutic implications. They provide a rationale for the traditional ammonia or indoles-lowering strategies, which have long been used in clinical management of HE. In addition, they point towards potential novel treatment strategies, including novel non-absorbable broad-spectrum antibiotics, and future microbial-specific antibiotics to avoid the common side effects of currently used antibiotics, towards drugs aimed at increased astrocyte activation in the brain (including minocycline), as well as different probiotic and prebiotic treatments. ${ }^{91} 95$ It is conceivable that the choice of therapeutic approach and the prediction of outcome will be influenced by the baseline assessment of gut microbial composition and metabolite profiles (box 5).

\section{CONCLUSIONS}

Microbiome research in liver disease has evolved recently as an exciting new research field. Clinical but especially preclinical

\section{Box 5 Gut-brain axis and microbiota}

- There are bidirectional communication channels between the gut, its microbiota and the brain

- The trafficking in these channels is significantly altered in hepatic encephalopathy (HE) due to alterations in the gut microbiota and their metabolites, as well as to increased permeability of the epithelial barrier and the blood-brain barrier

- In $\mathrm{HE}$, both inflammatory signal as well as neuroactive microbial metabolites reach the brain and can induce regional neuroinflammation in the brain

- Both structural and functional brain alterations have been reported in patients with $\mathrm{HE}$, and these alterations show correlations with behavioural alterations and symptoms

- Altered brain gut microbiome interactions in HE provide targets for novel treatment approaches, including prebiotics and probiotics, and microbe-specific antibiotics

experimental studies have suggested that microbial factors are driving forces in many different liver diseases and at various stages of liver diseases. The microbiota affects rather diverse pathophysiological processes such as hepatic steatosis, liver inflammation, fibrosis development and HE. These studies have now convincingly established that the microbiota play a critical role in these diseases. Nevertheless, more studies are needed, especially metagenomic and metabolomic studies are eagerly expected to first of all provide respective descriptive data on how the gut microbes and their metabolites are altered in various clinical settings of liver disease. In a second and more comprehensive step, preclinical studies are mandatory to settle which microbiotal strains affect disease phenotype, that is, either acting protective or detrimental. In a further clinical step, clinical studies will be needed in different clinical situations to manipulate the microbiota by various strategies including either prebiotics, new probiotics or antibiotics or using FMT. It needs to be established in the future how manipulation of the gut microbiota might prove beneficial for the treatment of patients with various liver diseases at either early or later disease stages. Anyway, a new area has started in hepatology, which will attract many scientists and physicians to investigate all these new excitements.

Twitter Follow Patrice Cani at @MicrObesity

Funding $\mathrm{HT}$ is supported by the excellence initiative (Competence Centers for Excellent Technologies) of the Austrian Research Promotion Agency (FFG): Research Center of Excellence in Vascular Ageing-Tyrol, VASCage (K-Project Nr. 843536) funded by the BMVIT, BMWFW, the Wirtschaftsagentur Wien and the

Standortagentur Tirol. PDC is a research associate at FRS-FNRS (Fonds de la Recherche Scientifique), Belgium. PDC is the recipient of grants from FNRS (J.0084.15) and ARC (Action de Recherche Concertée-Communauté française de Belgique convention: 12/17-047). This work was supported by WELBIO under grant: WELBIO-CR-2012S-02R, and the Funds Baillet Latour (Grant for Medical Research 2015). PDC is a recipient of an ERC Starting Grant 2013 (European Research Council, starting grant 336452-ENIGMO).

Competing interests None declared.

Provenance and peer review Commissioned; externally peer reviewed.

\section{REFERENCES}

1 Sender $R$, Fuchs $S$, Milo R. Are we really vastly outnumbered? Revisiting the ratio of bacterial to host cells in humans. Cell 2016;164:337-40.

2 Dominguez-Bello MG, Costello EK, Contreras M, et al. Delivery mode shapes the acquisition and structure of the initial microbiota across multiple body habitats in newborns. Proc Natl Acad Sci USA 2010;107:11971-5. 
3 Martin R, Nauta AJ, Ben Amor K, et al. Early life: gut microbiota and immune development in infancy. Benef Microbes 2010;1:367-82.

4 Cani PD, Everard A. Talking microbes: when gut bacteria interact with diet and host organs. Mol Nutr Food Res 2016;60:58-66.

5 Cotillard A, Kennedy SP, Kong LC, et al. Dietary intervention impact on gut microbial gene richness. Nature 2013;500:585-8.

6 Wu GD, Compher C, Chen EZ, et al. Comparative metabolomics in vegans and omnivores reveal constraints on diet-dependent gut microbiota metabolite production. Gut 2016;65:63-72.

7 Lim MY, Rho M, Song YM, et al. Stability of gut enterotypes in Korean monozygotic twins and their association with biomarkers and diet. Sci Rep 2014;4:7348.

8 Org E, Parks BW, Joo JW, et al. Genetic and environmental control of host-gut microbiota interactions. Genome Res 2015:25:1558-69.

9 Zeevi D, Korem T, Zmora N, et al. Personalized nutrition by prediction of glycemic responses. Cell 2015;163:1079-94.

10 David LA, Maurice CF, Carmody RN, et al. Diet rapidly and reproducibly alters the human gut microbiome. Nature 2014:505:559-63.

11 Everard A, Lazarevic V, Derrien $\mathrm{M}$, et al. Responses of gut microbiota and glucose and lipid metabolism to prebiotics in genetic obese and diet-induced leptin-resistant mice. Diabetes 2011;60:2775-86.

12 Rajilić-Stojanović M, Heilig HG, Molenaar D, et al. Development and application of the human intestinal tract chip, a phylogenetic microarray: analysis of universally conserved phylotypes in the abundant microbiota of young and elderly adults. Environ Microbiol 2009;11:1736-51.

13 Cani PD. Gut microbiota and obesity: lessons from the microbiome. Brief Funct Genomics 2013;12:381-7.

14 Blottière HM, de Vos WM, Ehrlich SD, et al. Human intestinal metagenomics: state of the art and future. Curr Opin Microbiol 2013;16:232-9.

15 Qin J, Li R, Raes J, et al. A human gut microbial gene catalogue established by metagenomic sequencing. Nature 2010;464:59-65.

16 Li J, Jia H, Cai X, et al. An integrated catalog of reference genes in the human gut microbiome. Nat Biotechnol 2014;32:834-41.

17 Sokol H, Pigneur B, Watterlot $L$, et al. Faecalibacterium prausnitzii is an anti-inflammatory commensal bacterium identified by gut microbiota analysis of Crohn disease patients. Proc Natl Acad Sci USA 2008;105:16731-6.

18 Everard A, Belzer C, Geurts L, et al. Cross-talk between Akkermansia muciniphila and intestinal epithelium controls diet-induced obesity. Proc Natl Acad Sci USA 2013;110:9066-71

19 Goodrich JK, Waters JL, Poole AC, et al. Human genetics shape the gut microbiome. Cell 2014;159:789-99.

20 Browne HP, Forster SC, Anonye BO, et al. Culturing of 'unculturable' human microbiota reveals novel taxa and extensive sporulation. Nature 2016;533:543-6.

21 Zoller $\mathrm{H}$, Tilg H. Nonalcoholic fatty liver disease and hepatocellular carcinoma. Metabolism 2016:65:1151-60.

22 Fiordaliso M, Kok N, Desager JP, et al. Dietary oligofructose lowers triglycerides, phospholipids and cholesterol in serum and very low density lipoproteins of rats. Lipids 1995;30:163-7.

23 Kok N, Roberfroid M, Delzenne N. Dietary oligofructose modifies the impact of fructose on hepatic triacylglycerol metabolism. Metabolism 1996;45:1547-50.

24 Delzenne NM, Kok N. Effect of non-digestible fermentable carbohydrates on hepatic fatty acid metabolism. Biochem Soc Trans 1998;26:228-30.

25 Daubioul CA, Taper HS, De Wispelaere LD, et al. Dietary oligofructose lessens hepatic steatosis, but does not prevent hypertriglyceridemia in obese Zucker rats. J Nutr 2000;130:1314-19.

26 Delzenne NM, Kok N. Effects of fructans-type prebiotics on lipid metabolism. Am J Clin Nutr 2001;73:456S-8S.

27 Letexier D, Diraison F, Beylot M. Addition of inulin to a moderately high-carbohydrate diet reduces hepatic lipogenesis and plasma triacylglycero concentrations in humans. Am J Clin Nutr 2003:77:559-64.

28 Roberfroid M, Gibson GR, Hoyles L, et al. Prebiotic effects: metabolic and health benefits. Br J Nutr 2010;104(Suppl 2):S1-S63.

29 Bindels LB, Porporato $\mathrm{P}$, Dewulf EM, et al. Gut microbiota-derived propionate reduces cancer cell proliferation in the liver. Br J Cancer 2012;107:1337-44.

30 Everard A, Lazarevic V, Gaïa N, et al. Microbiome of prebiotic-treated mice reveals novel targets involved in host response during obesity. ISME J 2014;8:2116-30.

31 Lin Y, Vonk RJ, Slooff MJ, et al. Differences in propionate-induced inhibition of cholesterol and triacylglycerol synthesis between human and rat hepatocytes in primary culture. Br J Nutr 1995;74:197-207.

32 Demigné $C$, Morand C, Levrat MA, et al. Effect of propionate on fatty acid and cholesterol synthesis and on acetate metabolism in isolated rat hepatocytes. $\mathrm{Br}$ J Nutr 1995:74:209-19.

33 Daubioul CA, Horsmans Y, Lambert $P$, et al. Effects of oligofructose on glucose and lipid metabolism in patients with nonalcoholic steatohepatitis: results of a pilot study. Eur J Clin Nutr 2005:59:723-6.

34 Malaguarnera $M$, Vacante $M$, Antic $T$, et al. Bifidobacterium longum with fructo-oligosaccharides in patients with non alcoholic steatohepatitis. Dig Dis Sci 2012;57:545-53.
35 Bäckhed F, Ding $H$, Wang $T$, et al. The gut microbiota as an environmental factor that regulates fat storage. Proc Natl Acad Sci USA 2004:101:15718-23.

36 Le Roy $\mathrm{T}$, Llopis $\mathrm{M}$, Lepage $\mathrm{P}$, et al. Intestinal microbiota determines development of non-alcoholic fatty liver disease in mice. Gut 2013:62:1787-94.

37 Raman M, Ahmed I, Gillevet PM, et al. Fecal microbiome and volatile organic compound metabolome in obese humans with nonalcoholic fatty liver disease. Clin Gastroenterol Hepatol 2013;11:868-75.e1-3.

38 Abdou RM, Zhu L, Baker RD, et al. Gut microbiota of nonalcoholic fatty liver disease. Dig Dis Sci 2016;61:1268-81.

39 Qin J, Li Y, Cai Z, et al. A metagenome-wide association study of gut microbiota in type 2 diabetes. Nature 2012;490:55-60.

40 Le Chatelier E, Nielsen T, Qin J, et al. Richness of human gut microbiome correlates with metabolic markers. Nature 2013:500:541-6.

41 Forslund $\mathrm{K}$, Hildebrand F, Nielsen T, et al. Disentangling type 2 diabetes and metformin treatment signatures in the human gut microbiota. Nature 2015;528:262-6.

42 Perry RJ, Peng L, Barry NA, et al. Acetate mediates a microbiome-brain- $\beta$-cell axis to promote metabolic syndrome. Nature 2016;534:213-17.

43 Pedersen HK, Gudmundsdottir V, Nielsen HB, et al. Human gut microbes impact host serum metabolome and insulin sensitivity. Nature 2016;535:376-81.

44 Dao MC, Everard A, Aron-Wisnewsky J, et al. Akkermansia muciniphila and improved metabolic health during a dietary intervention in obesity: relationship with gut microbiome richness and ecology. Gut 2016;65:426-36.

45 Cani PD, Amar J, Iglesias MA, et al. Metabolic endotoxemia initiates obesity and insulin resistance. Diabetes 2007;56:1761-72.

46 Cani PD, Bibiloni R, Knauf $C$, et al. Changes in gut microbiota control metabolic endotoxemia-induced inflammation in high-fat diet-induced obesity and diabetes in mice. Diabetes 2008;57:1470-81.

47 Cani PD, Possemiers S, Van de Wiele T, et al. Changes in gut microbiota control inflammation in obese mice through a mechanism involving GLP-2-driven improvement of gut permeability. Gut 2009;58:1091-103.

48 Henao-Mejia J, Elinav E, Jin C, et al. Inflammasome-mediated dysbiosis regulates progression of NAFLD and obesity. Nature 2012;482:179-85.

49 Xiao J, Tipoe GL. Inflammasomes in non-alcoholic fatty liver disease. Front Biosci (Landmark Ed) 2016:21:683-95.

50 Duparc T, Plovier H, Marrachelli VG, et al. Hepatocyte MyD88 affects bile acids, gut microbiota and metabolome contributing to regulate glucose and lipid metabolism. Gut Published Online First: 5 May 2016. doi:10.1136/gutjnl-2015-310904

51 Reid DT, McDonald B, Khalid T, et al. Unique microbial-derived volatile organic compounds in portal venous circulation in murine non-alcoholic fatty liver disease. Biochim Biophys Acta 2016;1862:1337-44.

52 Macnaughtan J, Jalan R. Clinical and pathophysiological consequences of alterations in the microbiome in cirrhosis. Am J Gastroenterol 2015;110:1399-410; quiz 411.

53 Tilg H, Diehl AM. Cytokines in alcoholic and nonalcoholic steatohepatitis. N Engl J Med 2000;343:1467-76

54 Mutlu EA, Gillevet PM, Rangwala $\mathrm{H}$, et al. Colonic microbiome is altered in alcoholism. Am J Physiol Gastrointest Liver Physiol 2012;302:G966-78.

55 Leclercq S, Matamoros S, Cani PD, et al. Intestinal permeability, gut-bacteria dysbiosis, and behavioral markers of alcohol-dependence severity. Proc Natl Acad Sci USA 2014;111:E4485-93.

56 Llopis M, Cassard AM, Wrzosek L, et al. Intestinal microbiota contributes to individual susceptibility to alcoholic liver disease. Gut 2016;65:830-9.

57 Chen $\mathrm{P}$, Miyamoto $\mathrm{Y}$, Mazagova M, et al. Microbiota protects mice against acute alcohol-induced liver injury. Alcohol Clin Exp Res 2015;39:2313-23.

58 Wang $L$, Fouts DE, Stärkel $P$, et al. Intestinal REG3 lectins protect against alcoholic steatohepatitis by reducing mucosa-associated microbiota and preventing bacterial translocation. Cell Host Microbe 2016;19:227-39.

59 Kirpich IA, Petrosino J, Ajami N, et al. Saturated and unsaturated dietary fats differentially modulate ethanol-induced changes in gut microbiome and metabolome in a mouse model of alcoholic liver disease. Am J Pathol 2016;186:765-76.

60 Neyrinck AM, Etxeberria U, Taminiau B, et al. Rhubarb extract prevents hepatic inflammation induced by acute alcohol intake, an effect related to the modulation of the gut microbiota. Mol Nutr Food Res Published Online First: May 2016. doi:10.1002/mnfr.201500899

61 Kummen M, Holm K, Anmarkrud JA, et al. The gut microbial profile in patients with primary sclerosing cholangitis is distinct from patients with ulcerative colitis without biliary disease and healthy controls. Gut Published Online First: 17 Feb 2016. doi:1136/gutjnl-2015-310500

62 Sabino J, Vieira-Silva S, Machiels K, et al. Primary sclerosing cholangitis is characterised by intestinal dysbiosis independent from IBD. Gut 2016;65:1681-9.

63 Bajaj JS, Hylemon PB, Ridlon JM, et al. Colonic mucosal microbiome differs from stool microbiome in cirrhosis and hepatic encephalopathy and is linked to cognition and inflammation. Am J Physiol Gastrointest Liver Physiol 2012;303:G675-85.

64 Torres J, Bao X, Goel A, et al. The features of mucosa-associated microbiota in primary sclerosing cholangitis. Aliment Pharmacol Ther 2016;43:790-801.

65 Tabibian JH, O'Hara SP, Trussoni CE, et al. Absence of the intestinal microbiota exacerbates hepatobiliary disease in a murine model of primary sclerosing cholangitis. Hepatology 2016;63:185-96. 
66 Wu X, Sun R, Chen Y, et al. Oral ampicillin inhibits liver regeneration by breaking hepatic innate immune tolerance normally maintained by gut commensal bacteria. Hepatology 2015;62:253-64.

67 Liu HX, Rocha CS, Dandekar S, et al. Functional analysis of the relationship between intestinal microbiota and the expression of hepatic genes and pathways during the course of liver regeneration. J Hepatol 2016;64:641-50.

68 Karin M, Clevers $\mathrm{H}$. Reparative inflammation takes charge of tissue regeneration. Nature 2016;529:307-15.

69 Chen Y, Yang F, Lu H, et al. Characterization of fecal microbial communities in patients with liver cirrhosis. Hepatology 2011;54:562-72.

70 Bajaj JS, Heuman DM, Hylemon PB, et al. Altered profile of human gut microbiome is associated with cirrhosis and its complications. J Hepatol 2014;60:940-7.

71 Bajaj JS, Betrapally NS, Hylemon PB, et al. Salivary microbiota reflects changes in gut microbiota in cirrhosis with hepatic encephalopathy. Hepatology 2015;62:1260-71.

72 Qin N, Yang F, Li A, et al. Alterations of the human gut microbiome in liver cirrhosis. Nature 2014;513:59-64.

73 Xie G, Wang $X$, Liu $P$, et al. Distinctly altered gut microbiota in the progression of liver disease. Oncotarget 2016;7:19355-66.

74 Mayer EA, Tillisch K, Gupta A. Gut/brain axis and the microbiota. J Clin Invest 2015;125:926-38.

75 Cryan JF, Dinan TG. Mind-altering microorganisms: the impact of the gut microbiota on brain and behaviour. Nat Rev Neurosci 2012;13:701-12.

76 Collins SM, Surette M, Bercik P. The interplay between the intestinal microbiota and the brain. Nat Rev Microbiol 2012;10:735-42.

77 Cani PD, Knauf C. How gut microbes talk to organs: the role of endocrine and nervous routes. Mol Metab 2016:5:743-52.

78 Mayer EA, Labus JS, Tillisch K, et al. Towards a systems view of IBS. Nat Rev Gastroenterol Hepatol 2015:12:592-605.

79 Mayer EA, Knight R, Mazmanian SK, et al. Gut microbes and the brain: paradigm shift in neuroscience. J Neurosci 2014;34:15490-6.

80 Butterworth RF. The liver-brain axis in liver failure: neuroinflammation and encephalopathy. Nat Rev Gastroenterol Hepatol 2013;10:522-8.

81 Rai R, Saraswat VA, Dhiman RK. Gut microbiota: its role in hepatic encephalopathy. J Clin Exp Hepatol 2015;5:S29-36.

82 Shawcross DL. Is it time to target gut dysbiosis and immune dysfunction in the therapy of hepatic encephalopathy? Expert Rev Gastroenterol Hepatol 2015;9:539-42.
83 Ahluwalia V, Betrapally NS, Hylemon PB, et al. Impaired gut-liver-brain axis in patients with cirrhosis. Sci Rep 2016;6:26800.

84 Shawcross DL, Olde Damink SW, Butterworth RF, et al. Ammonia and hepatic encephalopathy: the more things change, the more they remain the same. Metab Brain Dis 2005;20:169-79.

85 Romero-Gómez M, Jover M, Galán JJ, et al. Gut ammonia production and its modulation. Metab Brain Dis 2009;24:147-57.

86 Olde Damink SW, Jalan R, Deutz NE, et al. The kidney plays a major role in the hyperammonemia seen after simulated or actual $\mathrm{Gl}$ bleeding in patients with cirrhosis. Hepatology 2003;37:1277-85.

87 Ahboucha S, Butterworth RF. Role of endogenous benzodiazepine ligands and their GABA-A - associated receptors in hepatic encephalopathy. Metab Brain Dis 2005:20:425-37.

88 Baraldi M, Avallone R, Corsi L, et al. Natural endogenous ligands for benzodiazepine receptors in hepatic encephalopathy. Metab Brain Dis 2009;24:81-93.

89 Cauli 0 , Mansouri MT, Agusti A, et al. Hyperammonemia increases GABAergic tone in the cerebellum but decreases it in the rat cortex. Gastroenterology 2009;136:1359-67, e1-2.

90 Bravo JA, Forsythe $\mathrm{P}$, Chew MV, et al. Ingestion of Lactobacillus strain regulates emotional behavior and central GABA receptor expression in a mouse via the vagus nerve. Proc Natl Acad Sci USA 2011;108:16050-5.

91 Ahluwalia V, Wade JB, Heuman DM, et al. Enhancement of functional connectivity, working memory and inhibitory control on multi-modal brain MR imaging with rifaximin in cirrhosis: implications for the gut-liver-brain axis. Metab Brain Dis 2014;29:1017-25.

92 Kang DJ, Betrapally NS, Ghosh SA, et al. Gut microbiota drive the development of neuroinflammatory response in cirrhosis in mice. Hepatology 2016;64:1232-48.

93 Riggio O, Mannaioni G, Ridola L, et al. Peripheral and splanchnic indole and oxindole levels in cirrhotic patients: a study on the pathophysiology of hepatic encephalopathy. Am J Gastroenterol 2010;105:1374-81.

94 Montagnese S, Biancardi A, Schiff S, et al. Different biochemical correlates for different neuropsychiatric abnormalities in patients with cirrhosis. Hepatology 2011;53:558-66

95 Mittal VV, Sharma BC, Sharma P, et al. A randomized controlled trial comparing lactulose, probiotics, and L-ornithine L-aspartate in treatment of minimal hepatic encephalopathy. Eur J Gastroenterol Hepatol 2011;23:725-32. 